Article

\title{
Characterization of Fractional Polysaccharides from Gleditsia sinensis and Gleditsia microphylla Gums
}

\author{
Yantao Liu ${ }^{1}$, Zhenglong $X u^{1}$, Weian Zhang ${ }^{1}$, Jiufang Duan ${ }^{1}$, Jianxin Jiang ${ }^{1, *}$ and Dafeng Sun ${ }^{2, *}$ \\ 1 MOE Engineering Research Center of Forestry Biomass Materials and Bioenergy, Beijing Forestry University, \\ Beijing 100083, China; liuyt0321@163.com (Y.L.); zwa728@sina.com (Z.X.); ziyuanbeijing@163.com (W.Z.); \\ duanjiu99@163.com (J.D.) \\ 2 Nanjing Institute for the Comprehensive Utilization of Wild Plant, Nanjing 210042, China \\ * Correspondence: jiangjx2004@hotmail.com (J.J.); sdafeng@163.com (D.S.); \\ Tel.: +86-10-6233-8152 (J.J.); +86-25-8528-1405 (D.S.)
}

Academic Editors: Quan-Bin Han, Sunan Wang, Shaoping Nie and Derek J. McPhee Received: 10 October 2016; Accepted: 14 December 2016; Published: 19 December 2016

\begin{abstract}
The seeds of Gleditsia sinensis and Gleditsia microphylla, widespread in China, are an important source of galactomannans. G. sinensis gum (GSG) and G. microphylla gum (GMG) were purified and precipitated using different concentrations of ethanol and isopropanol. The GSG and GMG, precipitated in different stages, presented different characteristics, including polymer recovery, mannose/galactose ratio, chemical composition, molecular weight, and morphological appearance. The galactomannan recovery of GSG and GMG in 33.3\% ethanol was $81.7 \%$ and $82.5 \%$, respectively, while that in $28.8 \%$ isopropanol was $81.3 \%$ and $82.9 \%$, respectively. To achieve similar precipitation efficiency, the amount of isopropanol should be lower than that of ethanol because of the lower dielectric constant of isopropanol ( $20 \mathrm{vs.} 25$ for ethanol). The precipitation behavior of galactomannans in polar organic solvents was dependent on the molecular structures and properties of the solvent. A higher mannose/galactose ratio and a higher molecular weight was obtained in a lower concentration of alcohols.
\end{abstract}

Keywords: Gleditsia sinensis; Gleditsia microphylla; ethanol; isopropanol; fractionation

\section{Introduction}

Galactomannans are found in the endosperm of numerous plants, particularly the Leguminosae. Galactomannan gums are high-molecular-weight carbohydrates comprising a $\beta$-(1,4)-D-mannan backbone with single $\alpha-(1,6)$-linked D-galactose branches. Their mannose/galactose $(M / G)$ ratios and molecular mass distribution differ according to species [1].

Galactomannans can often be used in different forms for human consumption. They are excellent stiffeners and stabilizers of emulsions, and have wide applications in various industries, including the textile, pharmaceutical, biomedical, cosmetics, and food industries. However, most galactomannans used in pharmaceutical technology and cosmetics are usually unpurified gums [2,3].

Recently, alternative sources of seed gums have attracted increasing attention. The seeds of G. sinensis and G. microphylla (Leguminosae family) are two nontraditional sources of galactomannans. G. sinensis and G. microphylla are woody species that can grow in a wide range of environmental conditions. In China, they are widely distributed and are used as food, medicine, and health care products, among others [4-6].

Purification of these polymers can eliminate impurities and endogenous enzymes in coarse samples, improving their mass stability in the application process. Using ethanol and isopropanol $[7,8]$ as solvents to obtain these carbohydrates can promote intramolecular associations between water-soluble polymers through water competition. The dielectric constants of ethanol (20) and 
isopropanol (25) are much lower than that of water (80) at $25^{\circ} \mathrm{C}$ [9]. Therefore, adding ethanol and isopropanol can reduce the dielectric constant of the polysaccharide solution and induce conformational changes in the polymer, thus allowing molecules to aggregate and precipitate.

In many studies, alcohols and other organic solvents have been used to precipitate galactomannans or xyloglucan [10] from the water-extracted gum solution [7,11-13]. However, most of these studies have focused on one-time purification with high alcohol concentrations; only a few studies have focused on fractional purification and precipitate characterization of the polysaccharide gum. Precipitation is important for the purification and isolation of galactomannan gum; however, little research has been conducted on the relationship between the molecular structure of the polymer and its precipitation behavior in alcohol.

In the present work, two types of galactomannans (G. sinensis gum (GSG) and G. microphylla gum $(G M G)$ ) with different molecular mass distributions and $M / G$ ratios were fractionally precipitated in ethanol- and isopropanol-water solutions. Other studies have used solvents such as chloroform [14] and petroleum ether [15] to extract galactomannans, which are not authorized in the food industry. The characterizations of the galactomannan precipitates, as well as their $M / G$ ratios, monosaccharide composition, intrinsic viscosity, molecular mass distribution, ash content, and polymer recovery yields, were determined, thus providing useful information about the purification of galactomannan gums and the influence of the molecular structure on their behavior.

\section{Results and Discussion}

\subsection{Chemical Composition and Rheological Properties}

The chemical composition of the crude and purified gums GSG and GMG are listed in Table 1. The effect of the chemical composition on the crude and purified gums with different solvents and its concentration was significant $(p<0.05)$. The galactomannan contents of the crude GSG and GMG were $86.3 \%$ and $71.5 \%$ on the dry mass basis, respectively. The $M / G$ ratios obtained for crude GSG and GMG were 3.0 and 3.2, respectively, which are consistent with the previous literature values [16]. They were very similar to the commercial tara gum (3.0), which is a kind of galactomannan gum and is widely used as a thickening agent and stabilizer in food applications [17]. The protein and ash contents were found to decrease dramatically after purification by ethanol and isopropanol precipitation. This phenomenon was in agreement with reports by other researchers [18]. In addition, for both gums, the purity of the sample precipitated by isopropanol was a little higher than that obtained by ethanol.

Table 1. Chemical composition of the crude and purified G. sinensis gum (GSG) and G. microphylla gum (GMG), both in ethanol and isopropanol.

\begin{tabular}{|c|c|c|c|c|c|c|c|c|}
\hline Samples & Crude Gum & $\begin{array}{c}\text { Ethanol- } \\
\text { Purified GSG }{ }^{2}\end{array}$ & $\begin{array}{c}\text { Isopropanol- } \\
\text { Purified GSG }^{3} \\
\end{array}$ & $p$-Value & Crude GMG & $\begin{array}{c}\text { Ethanol- } \\
\text { Purified GSG }\end{array}$ & $\begin{array}{c}\text { Isopropanol- } \\
\text { Purified GSG }^{3} \\
\end{array}$ & $p$-Value \\
\hline Protein (\%) & $4.00 \pm 0.02^{1}$ & $0.96 \pm 0.03$ & $0.94 \pm 0.02$ & $<0.001$ & $7.41 \pm 0.06$ & $0.86 \pm 0.02$ & $0.76 \pm 0.02$ & $<0.001$ \\
\hline Ash (\%) & $0.74 \pm 0.09$ & $0.32 \pm 0.02$ & $0.20 \pm 0.02$ & 0.012 & $1.54 \pm 0.05$ & $0.38 \pm 0.03$ & $0.28 \pm 0.02$ & $<0.001$ \\
\hline$M / G^{4}$ & $3.0 \pm 0.03$ & $3.1 \pm 0.07$ & $3.4 \pm 0.06$ & 0.031 & $3.2 \pm 0.004$ & $2.9 \pm 0.07$ & $2.9 \pm 0.06$ & 0.044 \\
\hline
\end{tabular}

${ }^{1}$ Data are mean \pm SD of three determinations on a dry weight basis. ${ }^{2}$ Galactomannan gum purified by $33.3 \%$ ethanol precipitation. ${ }^{3}$ Galactomannan gum purified by $28.6 \%$ isopropanol precipitation. ${ }^{4} \mathrm{M} / \mathrm{G}$ : mannose/galactose ratio.

The flow curves of the $0.5 \%(w / w)$ aqueous solutions of GSG and GMG are shown in Figure 1. The apparent viscosity of the gum solution decreased and the shear stress increased with the increasing shear rate, indicating a typical non-Newtonian flow for the polymer $[19,20]$. For a solution to be considered Newtonian, its flow behavior index $(n)$ must be equal to 1 , while a value of $1>n>0$ indicates a pseudoplastic fluid behavior [21,22]. As shown in Table 2, the $n$ values of all the gum solutions were less than 1.00, suggesting that they were non-Newtonian fluids. The $n$ values of the $0.5 \%$ GSG and GMG solutions were 0.760 and 0.963 , respectively, which also suggests that the two galactomannan-type gums have a shear-thinning behavior, usually assigned to the pseudoplastic 
fluids [23,24]. The characteristic of the pseudoplastic fluid of galactomannan from GSG and GMG might be due to the entanglement interactions between the polymer chains. Therefore, at a lower shear rate, the longer molecular chains of GSG presented a random-coil conformation in aqueous solutions and had a correspondingly higher apparent viscosity, while, at a higher shear rate, the successively increasing shear stress could lead to the deformation or breakdown of intermolecular linkages and therefore a decline in the apparent viscosity was observed [25]. The $n$-values of guar gum (GG) and locust bean gum (LBG) solution were 0.571 and 0.834 [26], respectively, which means that GSG and LBG solutions are similar, implying that the GSG and LBG have similar shear stress and apparent viscosity. However, the $n$-value of the GMG solution was 0.963 , which was much higher than that of GSG, LBG, and GG. This difference of the rheological behavior of GSG and GMG may be because the galactomannan content and the molecular weight (from Table 2) of the crude polymer from G. microphylla was much lower than that of the crude from G. sinensis.
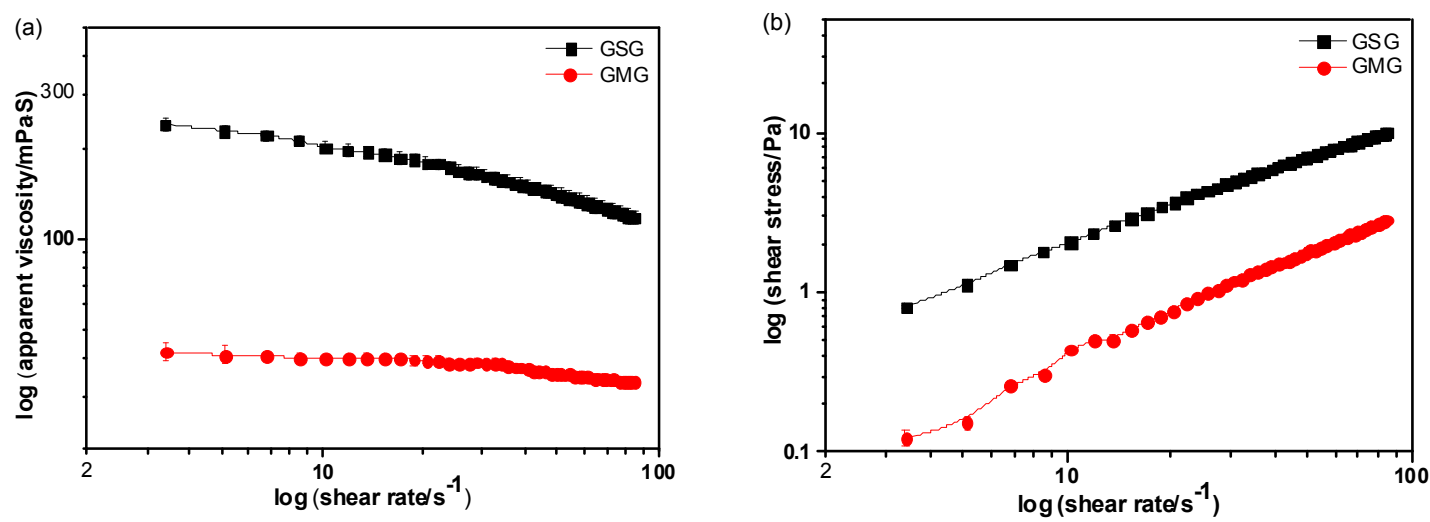

Figure 1. Flow curves of $0.5 \%(w / w)$ aqueous solutions of GSG (a) and GMG (b) at $30^{\circ} \mathrm{C}$.

Table 2. Rheological parameters of the crude GSG, GMG, GG, and LBG.

\begin{tabular}{ccccc}
\hline Power-Law Model & $\boldsymbol{k}$ & $\boldsymbol{n}$ & $\boldsymbol{R}^{\mathbf{2}}$ & References \\
\hline Crude GSG & 0.35 & 0.760 & 0.997 & \\
Crude GMG & 0.04 & 0.963 & 0.994 & \\
Crude GG $^{1}$ & 0.99 & 0.571 & 0.985 & {$[26]$} \\
Crude LBG $^{2}$ & 0.27 & 0.834 & 0.998 & {$[26]$} \\
\hline
\end{tabular}

${ }^{1}$ GG here means guar gum and ${ }^{2}$ LBG means locust bean gum. $R^{2}$ means correlation with the fitting equation (Equation (1), Section 3.2.2).

\subsection{Galactomannan Recovery and $M / G$ Ratio of the Precipitated Fractions}

The recovery yields and $M / G$ ratio of the galactomannan fractions for both polymers obtained by gradual alcohol precipitation are displayed in Figure 2; the calculation of the recovery yield (Equations (2)-(4), Section 3.2.4) was based on the galactomannan amount in the original crude gum dispersion. The total galactomannan recovery yields of GSG and GMG by ethanol precipitation (Figure 2a) were $81.7 \%$ and $82.5 \%$, while those obtained by isopropanol precipitation (Figure $2 \mathrm{~b}$ ) were $81.3 \%$ and $82.9 \%$, respectively, which do not seem to be statistically different. Even though it was evident that, to achieve a similar precipitation efficiency, the amount of ethanol used should be higher than that of isopropanol. The difference can be attributed to the lower dielectric constant of isopropanol. It can also be observed from Figure 2 that, during the fractionation process of both the ethanol and isopropanol precipitations, the recovery yield of galactomannan from GMG was always higher than that from GSG, except for the higher concentrations of the alcohols used. Perhaps the chemical differences between the two gums (GSG and GMG) can cause some changes during precipitation. One possible interpretation of these results is that, because the galactomannan content of 
crude GMG was lower than that of crude GSG, this allowed the galactomannan of GMG to precipitate more easily and promptly in the same concentration of both alcohols. It is well known that the solution properties of galactomannans are greatly affected by the degree and pattern of galactose substitution in galactomannan and that the solubility of galactomannans are directly related with the increasing $\mathrm{H}$-bonding density provided by the galactose side groups [27]. As shown in Figure 2, the M/G ratio obtained during the first stage of precipitation was higher than that of the corresponding crude gum (Table 1), the precipitation with the high molecular weight (Table 3), and the high value of M/G formed at low alcohol concentration. This might be explained by the fact that low-molecular-weight and highly substituted components are not as easily precipitated by ethanol or isopropanol as other solvents [28]. In another study, with the precipitation carried out in three stages, the molecular weight of the LBG decreased while its $M / G$ ratio increased with increasing alcohol concentration [26]. From Figure 2 and Table 3, we can see the precipitates of GSG and GMG formed in the first stage had the highest molecular weight (Table 3) and the highest $M / G$ ratio. The high molecular weight of both polymers obtained from GSG and GMG were less easily purified and precipitated in a low alcohol concentration.
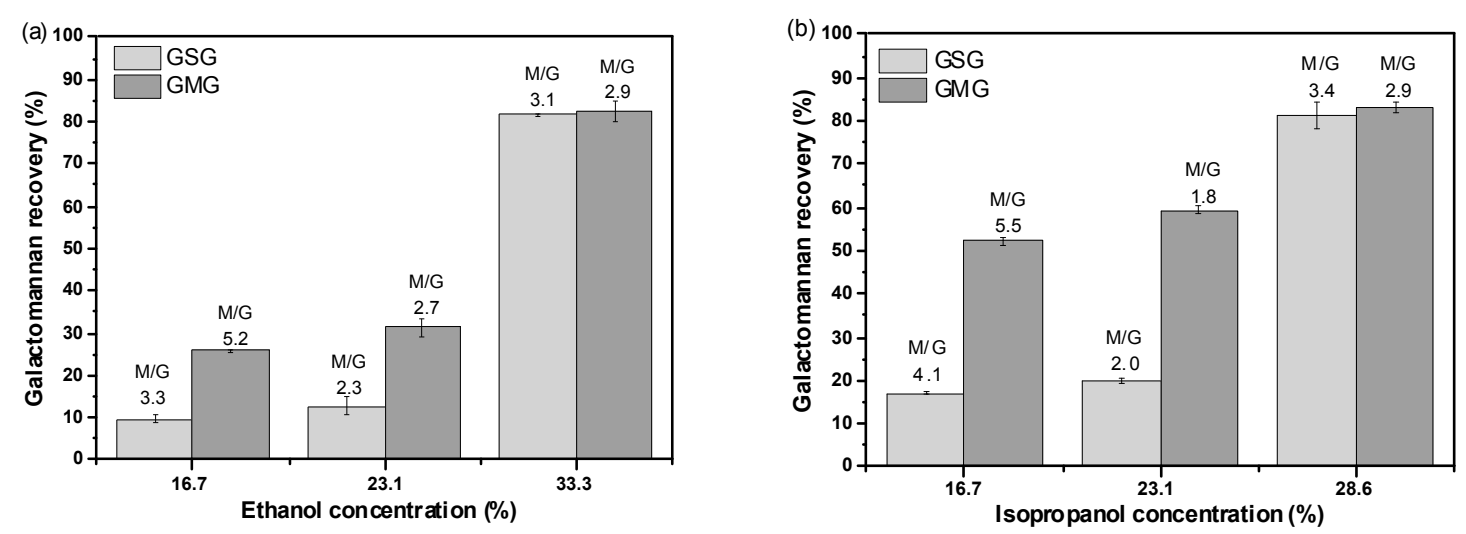

Figure 2. Galactomannan recovery yield and $M / G$ ratio of the gum fractions of GSG and GMG precipitated by (a) ethanol and (b) isopropanol. Numbers over the bars represent the M/G ratios.

Table 3. The $M_{w} M_{n}$ and $M_{w} / M_{n}$ of GSG and GMG during the ethanol/isopropanol fractional precipitation procedure.

\begin{tabular}{ccccccc}
\hline \multirow{2}{*}{ Samples } & \multicolumn{3}{c}{ GSG } \\
\cline { 2 - 7 } & $\mathbf{M}_{\mathbf{w}} / \mathbf{D a}$ & $\mathbf{M}_{\mathbf{n}} / \mathbf{D a}$ & $\mathbf{M}_{\mathbf{w}} / \mathbf{M}_{\mathbf{n}}$ & $\mathbf{M}_{\mathbf{w}} / \mathbf{D a}$ & $\mathbf{M}_{\mathbf{n}} / \mathbf{D a}$ & $\mathbf{M}_{\mathbf{w}} / \mathbf{M}_{\mathbf{n}}$ \\
\hline Crude gum & $7.865 \times 10^{5}$ & $5.042 \times 10^{5}$ & 1.56 & $6.245 \times 10^{5}$ & $5.027 \times 10^{5}$ & 1.24 \\
23.1\% Ethanol & $2.925 \times 10^{6}$ & $2.120 \times 10^{6}$ & 1.38 & $2.958 \times 10^{6}$ & $2.447 \times 10^{6}$ & 1.21 \\
28.6\% Ethanol & $3.466 \times 10^{5}$ & $2.611 \times 10^{5}$ & 1.33 & $4.007 \times 10^{5}$ & $3.710 \times 10^{5}$ & 1.08 \\
33.3\% Ethanol & $1.891 \times 10^{5}$ & $1.482 \times 10^{5}$ & 1.28 & $2.116 \times 10^{5}$ & $2.050 \times 10^{5}$ & 1.03 \\
16.7\% Isopropanol & $3.177 \times 10^{6}$ & $2.075 \times 10^{6}$ & 1.53 & $3.139 \times 10^{6}$ & $3.031 \times 10^{6}$ & 1.04 \\
23.1\% Isopropanol & $3.635 \times 10^{5}$ & $3.537 \times 10^{5}$ & 1.03 & $4.135 \times 10^{5}$ & $3.542 \times 10^{5}$ & 1.17 \\
28.6\% Isopropanol & $5.816 \times 10^{5}$ & $3.853 \times 10^{5}$ & 1.51 & $7.707 \times 10^{5}$ & $4.271 \times 10^{5}$ & 1.80 \\
\hline
\end{tabular}

\subsection{Fractional Precipitation by Ethanol and Isopropanol}

The wet weights of the three precipitated fractions of each gum was normalized to $100 \%$ (Equation (3), Section 3.2.4). The wet mass ratio and galactomannan concentration of each gel-like precipitate are summarized in Figure 3. In an ethanol solution (Figure 3a), the precipitates obtained with the highest alcohol concentration of the fractional precipitation procedure represented the largest proportion of the total precipitates; and, the similar results also obtained in the isopropanol solution (Figure 3b). With the increase in alcohol concentration in the bulk solution, the galactomannan content of the separated gel-like precipitate gradually increased, probably because of the increasing 
compactness of the precipitate [26]. The galactomannan concentration in the isopropanol-induced precipitate was significantly higher than that in the ethanol-induced precipitate because of the lower dielectric constant of isopropanol, resulting in a more compact precipitate than that found in ethanol. It can be concluded from Figure 3 that the high molecular weight (Table 3) and low galactomannan concentration of the precipitates formed at low ethanol/isopropanol concentrations were owing to the high levels of the entrained solvent in the gel-like precipitates; a further increase in the ethanol/isopropanol content increased the galactomannan concentration and purity [18,28]. At the same time, the morphological appearance of the ethanol/isopropanol precipitation had been observed in the first and third stages. In the first stage, precipitation from both alcohols formed a crude gum with a yellow, loose appearance; in the third stage, the alcohol/isopropanol precipitation formed a pure gum with a white, compact appearance. After the alcohol precipitation, especially the higher concentration of alcohol, the polysaccharide showed a marked increas in the purity with a bright whitish color.
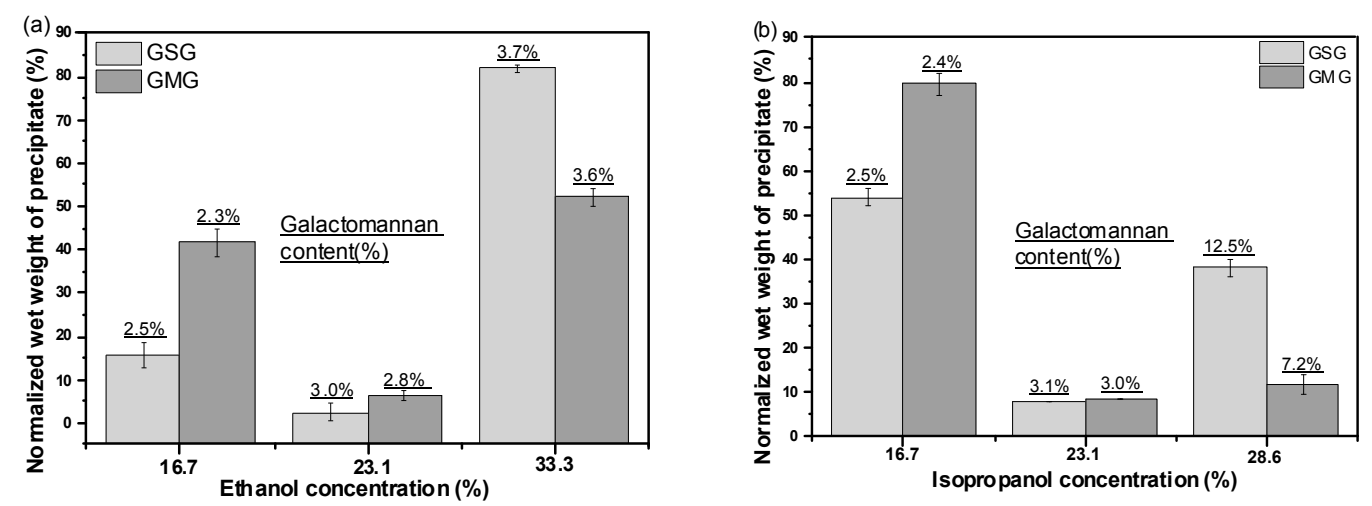

Figure 3. Normalized wet mass ratio and galactomannan concentration of the gum fractions of GSG and GMG obtained from (a) ethanol and (b) isopropanol precipitation.

\subsection{Molecular Weight}

The changes in the molecular-weight distribution in the ethanol and isopropanol fractionation process for GSG and GMG are shown in Table 3. It can be seen from Table 3 that the $\mathrm{M}_{\mathrm{w}}$ values for crude gum of GSG and GMG were $7.865 \times 10^{5}$ and $6.245 \times 10^{5} \mathrm{Da}$, respectively. This result of the $\mathrm{M}_{\mathrm{w}}$ values was consistent with the rheological properties of our previous measurements of the crude gum of GSG and GMG (as shown in Figure 1). After extracted with hot water of the crude gums, at lower alcohol concentrations, the molecular weight of the precipitated galactomannans was higher than those of the corresponding crude gums, indicating that the component of higher molecular weight was more prone to precipitation than those of lower molecular weight. This phenomenon may be due to the fact that the conformations of galactomannan fractions with different molecular weights might transform in water-alcohol solutions. Specifically, in an aqueous solution, polysaccharide molecules connect with water molecules through hydrogen bonds to maintain the solubility of the polysaccharide in water. Moreover, the addition of polar organic solvents such as ethanol and isopropanol would strengthen the intramolecular hydrogen bonding and induce conformational change and aggregation of polysaccharide molecules, eventually resulting in the precipitation of the molecule [29]. Consequently, adding ethanol or isopropanol to an aqueous solution of polysaccharide initially to obtain a certain concentration alcohol solution resulted in the precipitation of macromolecular chains, and with the increasing of alcohol added, the relatively low-molecular-weight fragments would precipitate, thus realizing the aim of fractional precipitation of polysaccharides on the basis of molecular structure. Therefore, it can be concluded that the conformational transition of galactomannans in polar ethanol and isopropanol is affected by the molecular structure (such as galactose substitution and molecular weight), and especially by inter- and intra-molecular hydrogen bonding. 


\section{Materials and Methods}

\subsection{Materials}

G. sinensis and G. microphylla seeds were supplied by Shexian Forestry Bureau in Hebei, China. The samples were collected in December 2012. They were manually separated and kept in a cool and dry place for further use. Moisture content of the whole seed was dried at $\left(105 \pm 2{ }^{\circ} \mathrm{C}\right)$ for constant weight $(10.0 \%(w / w))$. The standard monosaccharides (L-rhamnose, L-arabinose, D-glucose, D-galactose, D-mannose, and D-xylose) were purchased from Sigma-Aldrich (Saint Quentin Fallavier, France). Dextran standards (DXT3k, DXT25k, DXT160k, DXT760k, and DXT1185k) with an average molecular weight range of $3.7 \times 10^{3} \sim 1.2 \times 10^{6}$ Da were purchased from TosoHaas (Tokyo, Japan). Absolute ethanol and isopropanol were purchased from Beijing Chemical Works (Beijing, China). Calcium carbonate and concentrated sulfuric acid were all purchased from China National Pharmaceutical Group Corporation (Shanghai, China). Syringe filters were supplied by Tianjin branch billion Lung Experiment Equipment Co. Ltd., (Tianjin, China).

\subsection{Methods}

\subsubsection{Dehulling Pretreatment and Gum Preparation}

Baking treatment was used in dehulling pretreatment. About $30 \mathrm{~g}$ of seeds were put into the microwave oven (Galanz, G80F23DCN3L-F7(R0)) and baked for 6 min (just only use light wave mode $800 \mathrm{~W}$ ), followed by crushing during $3 \mathrm{~s}$ in a laboratory muller. After baking treatment, the hull was easily crushed into fragments, while the endosperm was harder crushing and almost unbroken in the crush. Most of the endosperm fragments were separated by sieving, the rest were manually separated. The endosperm was subsequently milled through a sieve size of $0.125 \mathrm{~mm}$. The two crude gums of GSG and GMG were obtained and subsequently were stored in a dryer for further use.

\subsubsection{Rheological Properties}

The rheological properties of the crude gums obtained from the seeds of G. sinensis and G. microphylla were carried out in a LVDV-III Ultra Rheometer (Brookfield Engineering Laboratories, Stoughton, MA, USA) equipped with a small sample adapter (SC13R) and spindle SC4-31. Samples for rheological measurement were prepared at a gum concentration of $0.5 \%(w / w)$ in distilled water on the dry weight basis. Then, the prepared gum solutions were placed in a water bath at $80^{\circ} \mathrm{C}$ for $30 \mathrm{~min}$ under magnetic stirring to produce uniform dispersion and complete hydration. The apparent viscosity of these crude gum solutions at $0.5 \%(w / w)$ were determined at $30{ }^{\circ} \mathrm{C}$ using exactly $9.0 \mathrm{~mL}$ of solution. All measurements were conducted at least in triplicate. Curves of the shear stress $(\sigma)$ as a function of the shear rate $(\gamma)$ for the two gum solutions were obtained by the software (Rheocalc V3.2, Stoughton, MA, USA) with the shear rate increasing from 1.7 to $85.0 \mathrm{~s}^{-1}$ within $4.5 \mathrm{~min}$.

$$
\sigma=\mathrm{k} \times \gamma^{\mathrm{n}}
$$

where $\mathrm{k}$ is the consistency index, and $\mathrm{n}$ is the flow index.

\subsubsection{Fractionation of the Crude Gums}

Two fractions for each of the gums from G. sinensis and G. microphylla were precipitated by the gradual addition of ethanol and isopropanol. First, however, the crude gum was dispersed into distilled water to achieve a gum concentration of $0.1 \%(w / w)$, and was then placed in a water bath at $80^{\circ} \mathrm{C}$ for $30 \mathrm{~min}$ with mechanical stirring. After full hydration, the gum dispersion was centrifuged at $12,000 \times g$ for $15 \mathrm{~min}$ to remove the insoluble residues, and the supernatant was collected for further fractional precipitation with ethanol and isopropanol. A certain volume of ethanol or isopropanol was added slowly to the supernatant under constant stirring to obtain a precipitate at room temperature 
$\left(25 \pm 3{ }^{\circ} \mathrm{C}\right)$. The resulting solution was centrifuged at $12,000 \times g$ for $15 \mathrm{~min}$ to separate the precipitate. The operation was repeated until no obvious precipitation was observed. All precipitates with the same concentration of alcohol were joined together. The wet precipitate was weighed and then dried in a vacuum oven for $6 \mathrm{~h}$ at $60{ }^{\circ} \mathrm{C}$ for constant weight [10]. The detailed fractionation procedure is summarized in Figure 4.

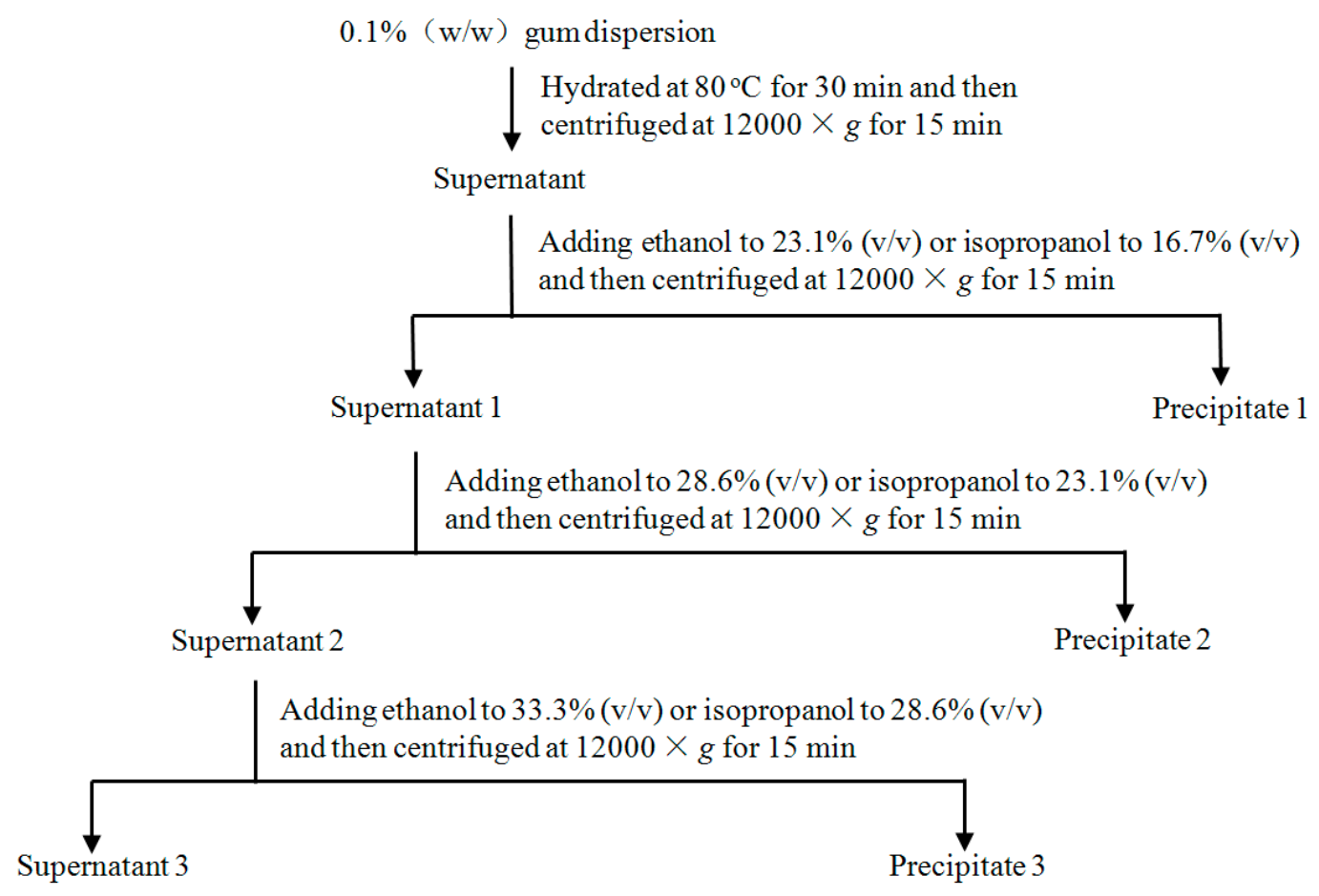

Figure 4. Scheme of the fractional precipitation of the two galactomannan gums of with ethanol and isopropanol.

During the fractional precipitation of the polysaccharides, the alcohol concentration in the alcohol-water solution was stepwise increased, and the formed polysaccharides precipitates were collected in each step, according to the method used by [26]. The precipitation experiment was carried out at least in triplicate.

\subsubsection{Chemical Composition of the Crude and Purified GSG and GMG}

The protein contents of G. sinensis and G. microphylla gums were performed according to the Kjeldahl method [30], after mineralization and distillation, and determined using a conversion factor of 6.25 [31]. The ash content of the gums was determined gravimetrically after dry mineralization at $550{ }^{\circ} \mathrm{C}$ for $5 \mathrm{~h}$ [31]. The galactomannan value of the gum samples was calculated, through the sum of mannose and galactose contents determined by HPLC monosaccharide analysis [7]. All the measurements were conducted at least in triplicate.

After a two-step hydrolysis with sulfuric acid, monosaccharide composition of the polysaccharide fraction was performed according to the NREL laboratory analytical procedure method [32]. In summary, the carbohydrate fraction was determined by hydrolyzing the gum sample ( $300 \mathrm{mg})$ with $3 \mathrm{~mL}$ of $72 \%(w / w) \mathrm{H}_{2} \mathrm{SO}_{4}$ for $1 \mathrm{~h}$ at $30{ }^{\circ} \mathrm{C}$ in a pressure tube with occasional vibration. Then, $84 \mathrm{~mL}$ of distilled water was added into the tube to achieve an acid concentration of $4.0 \%(w / w)$, and the sample was autoclaved for $1 \mathrm{~h}$ at $121^{\circ} \mathrm{C}$. After acid hydrolysis, the sample was neutralized with calcium carbonate. Hydrolyzed samples were filtered through a $0.22 \mu \mathrm{m}$ filter (Tianjin branch billion Lung Experiment Equipment Co. Ltd., Tianjin, China) and injected to a Waters 2695 HPLC system equipped with an Aminex HPX-87P column (300 mm $\times 7.8 \mathrm{~mm}$, Bio-Rad, Hercules, MA, USA) at 
$85^{\circ} \mathrm{C}$ and refractive index detector (2414 RID; Waters, Milford, MA, USA) at $35^{\circ} \mathrm{C}$. Double distilled water was used as the mobile phase with a flow rate of $0.6 \mathrm{~mL} / \mathrm{min}$. Calibration was performed with a series of standard sugar solutions of L-rhamnose, L-arabinose, D-glucose, D-galactose, D-mannose, and D-xylose.

The galactomannan recovery yield, the normalized weight percentage, and the polysaccharide content were calculated according to the following Equations:

$$
\begin{gathered}
\text { Recovery }(\%)=\frac{\sum_{i=1}^{n} \text { Galactomannan amount of Fraction } i(m g)}{\text { Galactomannan amount of crude gum }(m g)} \times 100 \\
\text { Normalized weight percent }(\%)=\frac{\text { wet weight of Fraction } i(m g)}{\text { total wet weight of all Fractions }(m g)} \times 100 \\
\text { Content }(\%)=\frac{\text { Galactomannan amount of Fraction } i(m g)}{\text { wet weight of Fraction } i(m g)} \times 100
\end{gathered}
$$

\subsubsection{Molecular Mass Determination}

The weight-average molecular weights $\left(\mathrm{M}_{\mathrm{w}}\right)$ of $\mathrm{G}$. sinensis and G. microphylla gums were determined by gel permeation chromatography (GPC) equipped with a refractive index detector and three columns in series, TSK PW XL guard column, TSK gel G $6000 \mathrm{PW}_{\mathrm{XL}}$ and TSK $\mathrm{Tel}_{\text {g }} 3000 \mathrm{PW} \mathrm{XL}_{\mathrm{L}}$ (TosoHaas, Tokyo, Japan). The mobile phase was a $0.2 \mathrm{M}$ phosphate buffer at $\mathrm{pH} 6.8$, with a flow rate of $0.6 \mathrm{~mL} / \mathrm{min}$. Samples were diluted to approximately $1.0 \mathrm{~g} / \mathrm{L}$ and filtered through a $0.45 \mathrm{~mm}$ filter (Tianjin branch billion Lung Experiment Equipment Co. Ltd. (Tianjin, China)) prior to injection onto the column. Dextran standards, with average molecular weights of $3.7 \times 10^{3} \sim 1.2 \times 10^{6} \mathrm{Da}$, were used to calibrate the columns, which were operated at $35{ }^{\circ} \mathrm{C}$ during measurements. The weight-average molecular weight $\left(M_{w}\right)$, the number-average molecular weight $\left(M_{n}\right)$, and $M_{w} / M_{n}$ were calculated with the Empower 2 software (Waters, Milford, MA, USA).

\subsection{Statistical Analysis}

The statistical significance of the experimental data was examined by analysis of variance (ANOVA) using SPSS version 17.0 (SPSS Inc., Armonk, NY, USA). The $p$-values were used as a tool to check the significant difference of the chemical compositions of the crude and purified gum. Values of $p$ less than $0.05(p<0.05)$ indicate a significant difference. A smaller $p$-value illustrates that the significance of the variable is more obvious.

\section{Conclusions}

GSG and GMG could be fractionated through gradual precipitation in ethanol and isopropanol. The purity of galactomannans precipitated by the ethanol and isopropanol precipitation was improved significantly and the property of a pseudoplastic solution was also confirmed in these two galactomannans. At low concentrations of alcohol, the formed precipitates were found to have a high mass ratio and low galactomannan concentration because of the high levels of the residual solvent. In addition, the concentration and purity of galactomannans increased with increasing alcohol concentration. To achieve the same precipitation efficiency, the amount of ethanol should be higher than that of isopropanol because of the low dielectric constant of isopropanol. In polar organic solvents, the precipitation behavior of galactomannans was related to the molecular structure such as galactose substitution and molecular weight, which was also closely linked to the aqueous solubility (hydration) of galactomannan.

Acknowledgments: The authors thank MOE Engineering Research Center of Forestry Biomass Materials and Bioenergy, Nanjing Institute for the Comprehensive Utilization of Wild Plant for providing all the necessary facilities to carry out this work. This research was financially supported by the China Ministry of Science and Technology (2016YFD0600803) and the National Natural Science Foundation of China (31270624, 31670579). 
Author Contributions: Yantao Liu and Zhenglong Xu conceived and designed the experiments; YanTao Liu, Zhenglong $\mathrm{Xu}$, and Weian Zhang performed the experiments; Yantao Liu, Weian Zhang, Jianxin Jiang, and Dafeng Sun analyzed the data; Zhenglong Xu and Jiufang Duan contributed reagents/materials/analysis tools; Yantao Liu and Weian Zhang wrote the paper.

Conflicts of Interest: The authors declare no conflict of interest.

\section{References}

1. Kok, M.S.; Hill, S.E.; Mitchell, J.R. Viscosity of galactomannans during high temperature processing: Influence of degradation and solubilisation. Food Hydrocoll. 1999, 13, 535-542. [CrossRef]

2. Brummer, Y.; Cui, W.; Wang, Q. Extraction, purification and physicochemical characterization of fenugreek gum. Food Hydrocoll. 2003, 17, 229-236. [CrossRef]

3. Uner, M.; Altinkurt, T. Evaluation of honey locust (Gleditsia triacanthos, Linn.) gum as sustaining material in tablet dosage forms. Farmaco 2004, 59, 567-573. [CrossRef] [PubMed]

4. Chow, L.M.C.; Tang, J.C.O.; Teo, I.T.N.; Chui, C.H.; Lau, F.Y.; Leung, T.W.T.; Cheng, G.; Wong, R.S.M. Antiproliferative Activity of the Extract of Gleditsia sinensis Fruit on Human Solid Tumour Cell Lines. Chemotherapy 2002, 48, 303-308. [CrossRef] [PubMed]

5. Chow, L.M.; Chui, C.H.; Tang, J.C. Gleditsia sinensis fruit extract is a potential chemotherapeutic agent in chronic and acute myelogenous leukemia. Oncol. Rep. 2003, 10, 1601-1607. [CrossRef] [PubMed]

6. Zhong, L.; Qu, G.; Li, P.; Han, J.; Guo, D. Induction of apoptosis and G2/M cell cycle arrest by Gleditsioside E from Gleditsia sinensis in HL-60 cell. Planta Med. 2003, 69, 561-563. [PubMed]

7. Jian, H.L.; Cristhian, C.; Zhang, W.M.; Jiang, J.X. Influence of dehulling pretreatment on physicochemical properties of Gleditsia sinensis Lam. gum. Food Hydrocoll. 2011, 25, 1337-1343. [CrossRef]

8. Navarro, D.A.; Cerezo, A.S.; Stortz, C.A. The 75\% Isopropanol-Soluble Polysaccharides from the Endosperm of the Legume Seed of Gleditsia triacanthos. Molecules 2000, 5, 543-544. [CrossRef]

9. Bilati, U.; Allemann, E.; Doelker, E. Development of a nanoprecipitation method intended for the entrapment of hydrophilic drugs into nanoparticles. Eur. J. Pharm. Sci. 2005, 24, 67-75. [CrossRef] [PubMed]

10. Chawananorasest, K.; Saengtongdee, P.; Kaemchantuek, P. Extraction and Characterization of Tamarind (Tamarind indica L.) Seed Polysaccharides (TSP) from Three Difference Sources. Molecules 2016, $21,775$. [CrossRef] [PubMed]

11. Amid, B.T.; Mirhosseini, H. Influence of different purification and drying methods on rheological properties and viscoelastic behaviour of durian seed gum. Carbohydr. Polym. 2012, 90, 452-461. [CrossRef] [PubMed]

12. Cunha, P.L.R.; Vieira, I.G.P.; Angela, A.M.C.; de Paula, R.C.M. Isolation and characterization of galactomannan from Dimorphandra gardneriana, Tul. seeds as a potential guar gum substitute. Food Hydrocoll. 2009, 23, 880-885. [CrossRef]

13. Icaro, G.P.V.; Gallao, M.I.; Brito, E.S.D. NMR study of galactomannans from the seeds of mesquite tree (Prosopis juliflora, (Sw) DC). Food Chem. 2007, 101, 70-73.

14. Mirzaeva, M.R.; Rakhmanberdyeva, R.K.E.L.; Kristallovich, R.D.A.; Shtonda, N.L. Water-soluble polysaccharides of seeds of the genus Gleditsia. Chem. Nat. Compd. 1998, 34, 653-655. [CrossRef]

15. Amin, A.M.; Ahmad, A.S.; Yin, Y.Y.; Yahya, N.; Ibrahim, N. Extraction, purification and characterization of durian (Durio zibethinus) seed gum. Food Hydrocoll. 2007, 21, 273-279. [CrossRef]

16. Jiang, J.X.; Jian, H.L.; Cristhian, C.; Zhang, W.M. Structural and thermal characterization of galactomannans from genus Gleditsia, seeds as potential food gum substitutes. J. Sci. Food Agric. 2011, 91, 732-737. [CrossRef] [PubMed]

17. Dakia, P.A.; Blecker, C.; Robert, C.; Wathelet, B.; Paquot, M. Composition and physicochemical properties of locust bean gum extracted from whole seeds by acid or water dehulling pre-treatment. Food Hydrocoll. 2008, 22, 807-818. [CrossRef]

18. Youssef, M.K.; Wang, Q.; Cui, S.W.; Barbut, S. Purification and partial physicochemical characteristics of protein free fenugreek gums. Food Hydrocoll. 2009, 23, 2049-2053. [CrossRef]

19. Wu, Y.; Cui, W.; Eskin, N.A.M.; Goff, H.D. An investigation of four commercial galactomannans on their emulsion and rheological properties. Food Res. Int. 2009, 42, 1141-1146. [CrossRef]

20. Tako, M.; Nakamura, S. Synergistic interaction between xanthan and guar gum. Carbohydr. Res. 1985, 138, 207-213. [CrossRef] 
21. Kayacier, A.; Dogan, M. Rheological properties of some gums-salep mixed solutions. J. Food Eng. 2006, 72, 261-265. [CrossRef]

22. Hosseiniparvar, S.H.; Matiamerino, L.; Goh, K.K.T.; Razavi, S.M.A.; Mortazavi, S.A. Steady shear flow behavior of gum extracted from Ocimum basilicum L. seed: Effect of concentration and temperature. J. Food Eng. 2010, 101, 236-243. [CrossRef]

23. Bourbon, A.I.; Pinheiro, A.C.; Ribeiro, C.; Miranda, C.; Maia, J.M.; Teixeira, J.A.; Vicente, A.A. Characterization of galactomannans extracted from seeds of Gleditsia triacanthos, and Sophora japonica, through shear and extensional rheology: Comparison with guar gum and locust bean gum. Food Hydrocoll. 2010, 24, 184-192. [CrossRef]

24. Alves, M.M.; Garnier, C.; Lefebvre, J.; Goncalves, M.P. Microstructure and flow behaviour of liquid water-gelatin-locust bean gum systems. Food Hydrocoll. 2001, 15, 117-125. [CrossRef]

25. Singh, V.; Sethi, R.; Tiwari, A. Structure elucidation and properties of a non-ionic galactomannan derived from the Cassia pleurocarpa seeds. Int. J. Biol. Macromol. 2009, 44, 9-13. [CrossRef] [PubMed]

26. Jian, H.L.; Lin, X.J.; Zhang, W.A.; Zhang, W.M.; Sun, D.F.; Jiang, J.X. Characterization of fractional precipitation behavior of galactomannan gums with ethanol and isopropanol. Food Hydrocoll. 2014, 40, 115-121. [CrossRef]

27. Pollard, M.A.; Fischer, P. Partial aqueous solubility of low-galactose-content galactomannans-What is the quantitative basis? Curr. Opin. Colloid Interface Sci. 2006, 11, 184-190. [CrossRef]

28. Silva, J.A.L.D.; Goncalves, M.P. Studies on a purification method for locust bean gum by precipitation with isopropanol. Food Hydrocoll. 1990, 4, 277-287. [CrossRef]

29. Lubambo, A.F.; Freitas, R.A.D.; Sierakowski, M.R. Electrospinning of commercial guar-gum: Effects of purification and filtration. Carbohydr. Polym. 2013, 93, 484-491. [CrossRef] [PubMed]

30. Bremner, J.M. Determination of nitrogen in soil by the Kjeldahl method. JAS 1960, 55, 11-33. [CrossRef]

31. Association of Official Analytical Chemists (AOAC) Standard. AOAC Official Methods of Analysis, 16th ed.; Washington, DC, USA, 1995.

32. Sluiter, A.; Hames, B.; Ruiz, R.; Scarlata, J.; Teplation, D.; Crocker, D. Determination of Structural Carbohydrates and Lignin in Biomass. Laboratory Analytical Procedure. Available online: http://www.nrel. gov/biomass/analytical_procedures.html (accessed on 3 August 2012).

Sample Availability: Samples of the compounds Gleditsia sinensis and Gleditsia microphylla gums are available from the authors.

(C) 2016 by the authors; licensee MDPI, Basel, Switzerland. This article is an open access article distributed under the terms and conditions of the Creative Commons Attribution (CC-BY) license (http://creativecommons.org/licenses/by/4.0/). 